\title{
COMPUTING STOCHASTIC DYNAMIC ECONOMIC MODELS WITH A LARGE NUMBER OF STATE VARIABLES: A DESCRIPTION AND APPLICATION OF A SMOLYAK-COLLOCATION METHOD
}

\author{
Benjamin Malin \\ Dirk Krueger \\ Felix Kubler \\ Working Paper 13517 \\ http://www.nber.org/papers/w13517 \\ NATIONAL BUREAU OF ECONOMIC RESEARCH \\ 1050 Massachusetts Avenue \\ Cambridge, MA 02138 \\ October 2007
}

This paper was prepared for the JEDC project on solving models with heterogeneous agents. We thank Ken Judd for clarifying discussions about the scope and focus of this paper and gratefully acknowledge financial support under NSF grant SES-0004376. The views expressed in this paper are solely our own and should not be interpreted as reflecting those of the Board of Governors, the staff of the Federal Reserve System, or the National Bureau of Economic Research.

(C) 2007 by Benjamin Malin, Dirk Krueger, and Felix Kubler. All rights reserved. Short sections of text, not to exceed two paragraphs, may be quoted without explicit permission provided that full credit, including (C) notice, is given to the source. 
Computing Stochastic Dynamic Economic Models with a Large Number of State Variables:

A Description and Application of a Smolyak-Collocation Method

Benjamin Malin, Dirk Krueger, and Felix Kubler

NBER Working Paper No. 13517

October 2007

JEL No. C68,C88,F41

\begin{abstract}
We describe a sparse grid collocation algorithm to compute recursive solutions of dynamic economies with a sizable number of state variables. We show how powerful this method may be in applications by computing the nonlinear recursive solution of an international real business cycle model with a substantial number of countries, complete insurance markets and frictions that impede frictionless international capital flows. In this economy the aggregate state vector includes the distribution of world capital across different countries as well as the exogenous country-specific technology shocks. We use the algorithm to efficiently solve models with 2, 4, and 6 countries (i.e., up to 12 continuous state variables).
\end{abstract}

Benjamin Malin

Federal Reserve Board of Governors

Mail Stop 97

21st and C St., NW

Washington, D.C. 20551

bmalin06@gmail.com

Dirk Krueger

Department of Economics

University of Pennsylvania

3718 Locust Walk

Philadelphia, PA 19104

and NBER

dkrueger@econ.upenn.edu
Felix Kubler

University of Pennsylvania

fkubler@gmail.com 


\title{
Computing Stochastic Dynamic Economic Models with a Large Number of State Variables: A Description and Application of a Smolyak-Collocation Method*
}

\author{
Benjamin Malin \\ Federal Reserve Board of Governors \\ Dirk Krueger \\ University of Pennsylvania, CEPR and NBER \\ Felix Kubler \\ University of Pennsylvania
}

September 28, 2007

\begin{abstract}
We describe a sparse grid collocation algorithm to compute recursive solutions of dynamic economies with a sizable number of state variables. We show how powerful this method may be in applications by computing the nonlinear recursive solution of an international real business cycle model with a substantial number of countries, complete insurance markets and frictions that impede frictionless international capital flows. In this economy the aggregate state vector includes the distribution of world capital across different countries as well as the exogenous country-specific technology shocks. We use the algorithm to efficiently solve models with 2,4 , and 6 countries (i.e., up to 12 continuous state variables).
\end{abstract}

\section{Introduction}

The stochastic neoclassical growth model has arguably been the most important workhorse in modern macroeconomic analysis. Its open economy counterpart, the international real business cycle model, has been fruitfully applied to study

*This paper was prepared for the JEDC project on solving models with heterogeneous agents. We thank Ken Judd for clarifying discussions about the scope and focus of this paper and gratefully acknowledge financial support under NSF grant SES-0004376. The views expressed in this paper are solely our own and should not be interpreted as reflecting those of the Board of Governors or the staff of the Federal Reserve System. 
the co-movement of output, investment, and consumption across countries and international capital flows between countries. See Backus et al. (1992) as an important example of this literature and Backus et al. (1995) for an overview.

Apart from highly stylistic examples, international real business cycles cannot be solved analytically, and one has to resort to numerical techniques to compute the equilibrium of these models. Even the simplest version of the model with two countries and country-specific, serially correlated technology shocks requires at least three state variables in its recursive formulation. This explains why most of the literature resorts to (log-)linearization of the equilibrium or optimality conditions of a world social planner problem to solve for equilibrium allocations. Since the true equilibrium is unknown, it is hard to assess how accurate numerical approximations of the equilibrium are that rely on these (log-)linear approximations.

In this paper we propose a projection method based on Smolyak's (1963) algorithm to compute globally accurate solutions to models characterized by a sizeable number of continuous state variables, such as international real business cycle models with a substantial number of countries. While collocation methods using full grids become infeasible for three or more dimensions, the use of a sparse grid constructed with Smolyak's algorithm allows us to handle ten or more state variables.

Our objectives are threefold: first, we provide an easily accessible general description of our algorithm; second, we show how powerful this method is by numerically solving an international real business cycle model with many countries and international capital market frictions; and third, we assess the quality of our approximations.

We find that our projection method performs quite well for a wide variety of model specifications including models with up to 6 countries (i.e., 12 continuous state variables), specifications that introduce a great deal of curvature into utility and production functions, and models with asymmetries between countries. Our method is also substantially more accurate than a linear approximation of the solution, especially when the exogenous shocks to the economy are large. The greatest challenge to using this method is finding bounds for the state variables which are not violated by the solution of the model. For international real business cycle models, this is most difficult for asymmetric specifications with a large number of countries.

The rest of the paper is organized as follows. Section 2 describes the international real business cycle model that we solve in this paper, while section 3 provides a general description of our projection method. Section 4 presents results, accuracy tests, and running times. Section 5 concludes.

\section{An International Real Business Cycle Model}

In this section, we introduce a simple dynamic model with a significant number of continuous state variables, an international real business cycle model with $N$ countries. We will confine ourselves to economies in which equilibria are Pareto 
optimal, so they can be characterized by solving an appropriately defined social planner's problem. In addition, we allow for frictions to international capital flows due to adjustment costs but require that these costs leave the optimal decision rules sufficiently smooth (that is, continuously differentiable).

\subsection{Description of the Model}

We consider a world composed of $N$ countries that are subject to technology shocks which contain a country-specific and a common component. Changes in a country's capital stock are subject to quadratic adjustment costs, which inhibit frictionless flows of capital across the $N$ countries. ${ }^{1}$ As a consequence the entire distribution of capital stocks, and not only aggregate output, becomes a state variable in the recursive formulation of the social planner's problem associated with this economy. There are complete asset markets, so that the welfare theorems apply. Thus one can solve for competitive equilibrium allocations by solving a social planner's problem for appropriate welfare weights of individual countries.

For a given set of Pareto weights $\left(\tau^{1}, \ldots, \tau^{N}\right)$, the social planner solves the problem

$$
\begin{aligned}
& \max _{\left.\left\{c_{t}^{i}, l_{t}^{i}, k_{t+1}^{i}\right\}_{i=1}^{N}\right\}_{t=0}^{\infty}} E_{0} \sum_{i=1}^{N} \tau^{i} \sum_{t} \beta^{t} u^{i}\left(c_{t}^{i}, l_{t}^{i}\right) \\
& \text { s.t. } \rho \ln \left(a_{t-1}^{i}\right)+\sigma\left(\varepsilon_{t}^{i}+\varepsilon_{t}\right) \\
& \ln \left(a_{t}^{i}\right)=(1-\delta) k_{t}^{i}+i_{t}^{i} \\
& k_{t+1}^{i}={ }^{N}= \sum_{i=1}^{N}\left(a_{t}^{i} f^{i}\left(k_{t}^{i}, l_{t}^{i}\right)-\frac{\phi}{2} \frac{\left(k_{t+1}^{i}-k_{t}^{i}\right)^{2}}{k_{t}^{i}}\right) \\
& \sum_{i=1}^{N} c_{t}^{i}+\sum_{i=1}^{N} i_{t}^{i}-\sum_{i=1}^{N} \delta k_{t}^{i}=
\end{aligned}
$$

where $\varepsilon_{t}, \varepsilon_{t}^{i}$ are iid standard normal random variables ${ }^{2}$ and $\phi \geq 0$ is a scale parameter. In particular, the parametrization nests the case of no adjustment costs, $\phi=0$. Denote the initial distribution of capital across countries by $\left(k_{0}^{1}, \ldots, k_{0}^{N}\right)$, which determines what point on the Pareto frontier (i.e. what vector of Pareto weights) corresponds to a competitive equilibrium. Our algorithm will solve for optimal policies for arbitrary Pareto weights, and thus for the entire equilibrium manifold. ${ }^{3}$

In general, the state variables for the recursive formulation of the social planner's problem consist of the vector of current exogenous shocks $a=\left(a^{1}, \ldots, a^{N}\right)$ and the vector of endogenous current capital stocks $k=\left(k^{1}, \ldots, k^{N}\right)$. Denote

\footnotetext{
${ }^{1}$ The quadratic form of the adjustment cost is not crucial for our algorithm to work. Any strictly convex and continuously differentiable adjustment cost function will do.

${ }^{2} \mathrm{As}$ long as we know the joint distribution of $\left(a_{t}^{1}, \ldots a_{t}^{N}\right)$ given $\left(a_{t-1}^{1}, \ldots a_{t-1}^{N}\right)$, we can numerically solve this model.

${ }^{3}$ Indirectly, and if we do the additional step of mapping Pareto weights into initial wealth distribution, directly.
} 
by $s=(k, a)$ the current state, which is of dimension $2 N$. Below we discuss one example where the number of state variables can be reduced to $N+1$.

The planner's problem in recursive formulation can be written as

$$
\begin{aligned}
V(k, a) & =\max _{\left\{c^{i}, l^{i}, k^{i \prime}\right\}} \sum_{i=1}^{N} \tau^{i} u^{i}\left(c^{i}, l^{i}\right)+\beta \int V\left(k^{\prime}, a^{\prime}\right) g_{a}\left(a^{\prime}\right) d a^{\prime} \\
\ln \left(a^{i \prime}\right) & =\rho \ln \left(a^{i}\right)+\sigma\left(\varepsilon_{i}+\varepsilon\right) \\
\sum_{i=1}^{N} c^{i}+\sum_{i=1}^{N} k^{i \prime}+\sum_{i=1}^{N} \frac{\phi\left(k^{i \prime}-k^{i}\right)^{2}}{2 k^{i}} & =\sum_{i=1}^{N} a^{i} f^{i}\left(k^{i}, l^{i}\right)+\sum_{i=1}^{N} k^{i}
\end{aligned}
$$

where $g_{a}\left(a^{\prime}\right)$ denotes the probability density function over $a^{\prime}$, given $a$. We will now derive the system of functional equations used to compute this model. We seek functions $C^{i}(s), L^{i}(s)$, and $K^{i \prime}(s)$ for $i=1, \ldots, N$, mapping the current state $s=(k, a)$ into consumption and labor supply of each country today and its capital stock tomorrow. For future reference we define as

$$
\begin{aligned}
C(s) & =\sum_{i=1}^{N} C^{i}(s) \\
Y(s) & =\sum_{i=1}^{N} a^{i} f^{i}\left(k^{i}, L^{i}(s)\right) \\
K & =\sum_{i=1}^{N} k^{i} \\
K^{\prime}(s) & =\left(K^{1 \prime}(s), \ldots, K^{N^{\prime}}(s)\right) .
\end{aligned}
$$

Attaching Lagrange multiplier $\mu$ to the resource constraint, we find as first order conditions

$$
\begin{array}{rlrl}
\tau^{i} u_{c}^{i}\left(c^{i}, l^{i}\right) & = & \mu & \forall i \\
\frac{\tau^{i} u_{l}^{i}\left(c^{i}, l^{i}\right)}{-a^{i} f_{l}^{i}\left(k^{i}, l^{i}\right)} & = & \mu & \forall i \\
\frac{\beta \int V_{k^{i}}\left(k^{\prime}, a^{\prime}\right) g_{a}\left(a^{\prime}\right) d a^{\prime}}{1+\frac{\phi\left(k^{i \prime}-k^{i}\right)}{k^{i}}} & =\mu & \forall i
\end{array}
$$

where lower case letters attached to functions denote partial derivatives of the function with respect to the corresponding argument. The envelope condition 
reads as

$$
\begin{array}{rlrl}
V_{k^{i}}(k, a) & =\mu\left[\left(1+a^{i} f_{k}^{i}\left(k^{i}, l^{i}\right)+\frac{\phi}{2} \frac{\left(k^{i \prime}-k^{i}\right)\left(k^{i \prime}+k^{i}\right)}{k^{i^{2}}}\right]\right. & \\
& =\tau^{i} u_{c}^{i}\left(c^{i}, l^{i}\right)\left[\left(1+a^{i} f_{k}^{i}\left(k^{i}, l^{i}\right)+\frac{\phi}{2} \frac{\left(k^{i \prime}-k^{i}\right)\left(k^{i \prime}+k^{i}\right)}{k^{i^{2}}}\right] \quad \forall i\right. \\
& =\tau^{j} u_{c}^{j}\left(c^{j}, l^{j}\right)\left[\left(1+a^{i} f_{k}^{i}\left(k^{i}, l^{i}\right)+\frac{\phi}{2} \frac{\left(k^{i \prime}-k^{i}\right)\left(k^{i \prime}+k^{i}\right)}{k^{i^{2}}}\right]\right. & \forall i, j .
\end{array}
$$

Combining the first order conditions and the envelope conditions gives (replacing choices by policy functions and abusing notation by writing $\left.s^{\prime}=\left(K^{\prime}(s), a^{\prime}\right)\right)$

$$
\begin{aligned}
& \tau^{i} u_{c}^{i}\left(C^{i}(s), L^{i}(s)\right)= \\
& \frac{\beta \int \tau^{i} u_{c}^{i}\left(C^{i}\left(s^{\prime}\right), L^{i}\left(s^{\prime}\right)\right)\left[\begin{array}{c}
1+a^{i \prime} f_{k}^{i}\left(K^{i \prime}, L^{i}\left(s^{\prime}\right)\right)+ \\
\frac{\phi}{2} \frac{\left(K^{i \prime}\left(s^{\prime}\right)-K^{i \prime}(s)\right)\left(K^{i \prime}\left(s^{\prime}\right)+K^{i \prime}(s)\right)}{K^{i \prime}(s)^{2}}
\end{array}\right] g_{a}\left(a^{\prime}\right) d a^{\prime}}{1+\frac{\phi\left(K^{i \prime}\left(s^{\prime}\right)-K^{i \prime}(s)\right)}{K^{i \prime}(s)}}
\end{aligned}
$$

which together with

$$
\begin{aligned}
\tau^{i} u_{c}^{i}\left(C^{i}(s), L^{i}(s)\right) & =\tau^{j} u_{c}^{j}\left(C^{j}(s), L^{j}(s)\right) \\
\frac{u_{l}^{i}\left(C^{i}(s), L^{i}(s)\right)}{u_{c}^{i}\left(C^{i}(s), L^{i}(s)\right)} & =-a^{i} f_{l}^{i}\left(k^{i}, L^{i}(s)\right) \\
C(s)+\sum_{i=1}^{N} K^{i \prime}(s)+\sum_{i=1}^{N} \frac{\phi\left(K^{i \prime}(s)-k^{i}\right)^{2}}{2 k^{i}} & =Y(s)+K
\end{aligned}
$$

provide $3 N$ functional equations to be jointly solved for the $3 N$ functions $\left\{C^{i}(s)\right.$, $\left.L^{i}(s), K^{i \prime}(s)\right\}_{i=1}^{N}$.

\subsection{Special Cases}

In general, these $3 N$ functional equations have to be solved jointly, but there are special cases where the problem becomes easier. If labor is supplied inelastically, we can drop the $N$ functional equations (3), leaving $2 N$ functional equations to be jointly solved for the $2 N$ functions $\left\{C^{i}(s), K^{i \prime}(s)\right\}_{i=1}^{N}$. There is a sense in which production and consumption decisions are separable. The $N-1$ equations

$$
\begin{gathered}
\beta \int \tau^{i} u_{c}^{i}\left(C^{i}\left(s^{\prime}\right)\right)\left[\begin{array}{c}
1+a^{i \prime} f_{k}^{i}\left(K^{i \prime}(s)\right)+ \\
\frac{\phi}{2} \frac{\left(K^{i \prime}\left(s^{\prime}\right)-K^{i \prime}(s)\right)\left(K^{i \prime}\left(s^{\prime}\right)+K^{i \prime}(s)\right)}{K^{\prime \prime}(s)^{2}}
\end{array}\right] g_{a}\left(a^{\prime}\right) d a^{\prime} \\
1+\frac{\phi\left(K^{i \prime}\left(s^{\prime}\right)-K^{i \prime}(s)\right)}{K^{i \prime}(s)} \\
=\frac{\beta \int \tau^{i} u_{c}^{i}\left(C^{i}\left(s^{\prime}\right)\right)\left[\begin{array}{c}
1+a^{j \prime} f_{k}^{j}\left(K^{i \prime}(s)\right)+ \\
\frac{\phi}{2} \frac{\left(K^{j \prime}\left(s^{\prime}\right)-K^{\prime \prime}(s)\right)\left(K^{j}\left(s^{\prime}\right)+K^{j^{\prime}}(s)\right)}{K^{\prime \prime}()^{2}}
\end{array}\right] g_{a}\left(a^{\prime}\right) d a^{\prime}}{1+\frac{\phi\left(K^{j^{\prime}}\left(s^{\prime}\right)-K^{j^{\prime}}(s)\right)}{K^{j^{\prime}}(s)}}
\end{gathered}
$$


for a given total amount of capital to be carried forward to tomorrow determine the allocation of capital across the countries. However, the decision of how much total consumption and how much accumulation is optimal cannot in general be solved for independent of the allocation of consumption across countries, which is simply another way of saying that the $2 N$ equations have to solved jointly. Note that so far no assumptions about the functional form of $u^{i}, f^{i}$ and the equality of preference or technology parameters have been made.

\subsubsection{Exogenous Labor and CRRA Utility}

There are two interesting examples for which the problem with exogenous labor supply becomes even easier. Suppose all households have identical CRRA period utility function with risk aversion parameter $\gamma$. Then from

$$
\tau^{i} u_{c}^{i}\left(C^{i}(s)\right)=\tau^{j} u_{c}^{j}\left(C^{j}(s)\right)
$$

it follows that

$$
C^{i}(s)=\left(\frac{\tau^{i}}{\tau^{1}}\right)^{\frac{1}{\gamma}} C^{1}(s)
$$

and thus

$$
C(s)=\sum_{i=1}^{N} C^{i}(s)=\frac{C^{1}(s)}{\left(\tau^{1}\right)^{\frac{1}{\gamma}}} \sum_{i=1}^{N}\left(\tau^{i}\right)^{\frac{1}{\gamma}}
$$

and thus consumption follows the linear risk sharing rule

$$
C^{i}(s)=\frac{\left(\tau^{i}\right)^{\frac{1}{\gamma}}}{\sum_{i=1}^{N}\left(\tau^{i}\right)^{\frac{1}{\gamma}}} C(s) .
$$

That is, each agents' consumption is a constant fraction of aggregate consumption. For this example one can first jointly solve for aggregate consumption, aggregate investment and its allocation across countries, and then separately solve for the distribution of consumption across countries, according to (6). Using (6) in (5) yields the $N-1$ equations

$$
\begin{aligned}
& \int\left(C\left(s^{\prime}\right)\right)^{-\gamma}\left[\begin{array}{c}
1+a^{i \prime} f_{k}^{i}\left(K^{i \prime}(s)\right)+ \\
\left.\frac{\phi}{2} \frac{\left(K^{i \prime}\left(s^{\prime}\right)-K^{i \prime}(s)\right)\left(K^{i \prime}\left(s^{\prime}\right)+K^{i \prime}(s)\right)}{K^{i \prime}(s)^{2}}\right]
\end{array}\right] g_{a}\left(a^{\prime}\right) d a^{\prime} \\
& 1+\frac{\phi\left(K^{i \prime}\left(s^{\prime}\right)-K^{i \prime}(s)\right)}{K^{i \prime}(s)} \\
& =\frac{\int\left(C\left(s^{\prime}\right)\right)^{-\gamma}\left[\begin{array}{c}
1+a^{j \prime} f_{k}^{j}\left(K^{i \prime}(s)\right)+ \\
\frac{\phi}{2} \frac{\left(K^{j \prime}\left(s^{\prime}\right)-K^{j^{\prime}}(s)\right)\left(K^{j^{\prime}}\left(s^{\prime}\right)+K^{j^{\prime}}(s)\right)}{K^{j \prime}(s)^{2}}
\end{array}\right] g_{a}\left(a^{\prime}\right) d a^{\prime}}{1+\frac{\phi\left(K^{j^{\prime}}\left(s^{\prime}\right)-K^{j^{\prime}}(s)\right)}{K^{j^{\prime}}(s)}}
\end{aligned}
$$

which, together with the resource constraint (4) and an equation similar to (1) can be solved for the functions $\left(C(s), K^{1 \prime}(s), \ldots, K^{N^{\prime}}(s)\right)$. Now indeed a complete separation between production and consumption allocations arises. 
This separation reduces the computational burden for this case by significantly reducing the number of equations that have to be solved simultaneously. But it does not ease the curse of dimensionality since no reduction in the number of continuous state variables is possible in this case.

\subsubsection{Exogenous Labor and No Capital Adjustment Cost}

If there are no adjustment costs, that is, if $\phi=0$, then we can reduce the number of state variables from the $2 N$ variables $(k, a)$ to the $N+1$ variables $s=(y, a)$ where $y$ is total output in the current period.

$$
\begin{aligned}
V(s) & =\max _{\left\{c^{i}, k^{i}\right\}} \sum_{i=1}^{N} \tau^{i} u^{i}\left(c^{i}\right)+\beta \int V\left(s^{\prime}\right) g_{a}\left(a^{\prime}\right) d a^{\prime} \\
\sum_{i=1}^{N} c^{i}+\sum_{i=1}^{N} k^{i \prime} & =\sum_{i=1}^{N} a^{i} f^{i}\left(k^{i}\right)+\sum_{i=1}^{N} k^{i} \\
y^{\prime} & =\sum_{i=1}^{N} a^{i \prime} f^{i}\left(k^{i \prime}\right) .
\end{aligned}
$$

The first order and envelope conditions imply

$$
\mu=\tau^{i} u_{c}^{i}\left(c^{i}\right)=\beta \int\left(1+a^{i \prime} f_{k}^{i}\left(k^{i \prime}\right)\right) V_{y}\left(y^{\prime}\left(a^{\prime}\right), a^{\prime}\right) g_{a}\left(a^{\prime}\right) d a^{\prime}=V_{y}(s) .
$$

The $2 N$ functional equations determining the functions $\left.\left\{C^{i}(s), K^{i \prime}(s)\right)\right\}_{i \in I}$ are the same as in the general case (with $\phi=0$ ), but the policy functions are simply functions of the $N+1$ state variables $s=(y, a)$.

In this special case models with more countries can feasibly be computed since the addition of one country adds only one, rather than two, continuous state variables to the problem.

\section{A Sparse Grid Collocation Method}

We will solve for an approximate equilibrium of the international real business cycle model using a Smolyak collocation method. The basic idea of collocation methods is to approximate the functions $\left\{C^{i}(s), L^{i}(s), K^{i \prime}(s)\right\}_{i=1}^{N}$ by weighted sums of 'easier functions', e.g. by polynomials. In order to determine the unknown coefficients of the polynomials, we require that equations (1)-(4) hold exactly at finitely many points - the so-called collocation points. While collocation methods are routinely used in economics to solve non-linear dynamic models, our innovation is to use a Smolyak sparse grid method which allows us to consider fairly high-dimensional problems. The use of sparse grids is well established in numerical analysis (see e.g. Bungartz and Griebel (2004) for an overview) and was first introduced, as far as we know, to economics by Krueger and Kubler (2004). 
From a technical point of view, collocation methods pose three challenges. First, the state space is high-dimensional ${ }^{4}$ which means that the policy functions, which have to be approximated, are high-dimensional. Second, the conditional expectations in agents' Euler equations are high-dimensional integrals ${ }^{5}$ which have to be evaluated very frequently in the solution procedure. Lastly, one has to solve a rather large system of non-linear equations to obtain the unknown coefficients.

While the focus of this paper is on the first problem (the high dimensionality of the state space), we will also discuss the issues associated with the second problem and propose a solution based on monomial rules (see Judd (1998)). Regarding the third problem, we chose to use a simple time-iteration scheme rather than more efficient methods - we briefly discuss this issue at the end of this section.

\subsection{Smolyak Sparse Grids}

In order to motivate the choice of Smolyak points as collocation points, we consider the abstract problem of how to approximate the unknown policy function $f:[-1,1]^{d} \rightarrow \mathbb{R}$ by interpolating a finite number of known function values, i.e. we try to find a finite number of points $\mathcal{H} \subset[-1,1]^{d}$ and a function $\hat{f}$ such that given the points $\left(x_{i}, y_{i}=f\left(x_{i}\right)\right)$ with $x_{i} \in \mathcal{H}$, the function $\hat{f}$ satisfies $\hat{f}\left(x_{i}\right)=f\left(x_{i}\right)$ and such that $\hat{f}$ approximates $f$ well on its entire domain $[-1,1]^{d}$. Here $d$ is the dimension of the problem, in our application the dimension of the state space. The remaining questions are then how to choose the interpolation points $\mathcal{H}$ and how to choose the interpolating function $\hat{f}$.

Smolyak's (1963) method provides both sets of points as well as formulas for the approximating functions. To describe Smolyak's method, adopted to the problem of high-dimensional interpolation on sparse grids by Barthelmann et al. (2000), we start by defining a set of points in $[-1,1]^{d}$ which can be interpolated by polynomials of relatively low degree. Then we give a formula for the interpolating polynomial. This description is meant to be a more accessible version of the discussion in Krueger and Kubler (2004), which in turn follows Barthelmann et al. (2000).

Since we know from the structure of the economy that the true policy function $f$ is smooth, we use a multivariate polynomial to approximate it. In one dimension, it is well known that one can interpolate $n$ points by a univariate polynomial of degree $n-1$, i.e. by a polynomial with $n$ terms of the form

$$
p_{n-1}(x)=\sum_{j=1}^{n} \theta_{j} x^{j-1} .
$$

\footnotetext{
${ }^{4}$ As shown above, it consists, in general, of the capital stocks in all countries as well as all country-specific technology shocks.

${ }^{5}$ The dimension of the integral is equal to the number of countries, if each country is hit by one technology shock. If the shock has finite support, then the integral is replaced by a sum.
} 
In order to find the unknown $n$ coefficients $\left(\theta_{1}, \ldots, \theta_{n}\right)$, one can simply use the $n$ equations (which are linear in the unknowns)

$$
y_{i}=\sum_{j=1}^{n} \theta_{j}\left(x_{i}\right)^{j-1} \text { for } i=1, \ldots, n .
$$

It is now common in economics to use the orthogonal Chebychev polynomials to express the approximating function and write

$$
p_{n-1}(x)=\sum_{j=1}^{n} \theta_{j} T_{j}(x)
$$

where the Chebychev polynomials $T_{1}(),. T_{2}(),. \ldots$ can be evaluated recursively by $T_{1}(x)=1, T_{2}(x)=x$ and $T_{j+1}(x)=2 x T_{j}(x)-T_{j-1}(x)$. While (9) is just a different way to express the same function as in (8), the advantage of using Chebychev polynomials is, that since the $T_{j}$ are all orthogonal, the coefficients are smaller and the interpolation problem better conditioned (see Judd (1998)). Although we use Chebychev polynomials in our method, it is important to understand that Smolyak's algorithm in no way depends on the use of this particular set of polynomials.

While approximation of a function by interpolation is straightforward in one dimension, it is much more complicated in several dimensions. In particular, it is not true that with a polynomial of $n$ terms one can interpolate arbitrary $n$ points in higher dimensions (in fact, this is the exception). Moreover, it is not obvious how to optimally choose the collocation points in higher dimensions. Any rule to choose these points has to satisfy, in order to be of practical use for high-dimensional problems, that the number of interpolation points does not grow exponentially in the dimensionality of the problem.

The simplest approach to multi-variate interpolation is to span a rectangular grid with $n$ values in each dimension and use a tensor product of one-dimensional polynomials as a set of approximating functions. Thus one would approximate a $d$-dimensional function $f:[-1,1]^{d} \rightarrow \mathbb{R}$ by interpolating the function values at the $n^{d}$ grid points by a polynomial of total degree ${ }^{6}(n-1) d$. The problem with this approach is that it becomes infeasible very fast as $d$ becomes large since the number of unknown coefficients grows exponentially with the dimension $d$. If one chooses $n^{d}$ points and thus univariate polynomials of degree $n-1$, the

\footnotetext{
${ }^{6}$ The degree of a multi-variate polynomial is defined to be the degree of its leading monomial. If the set of one-dimensional polynomials of degree $n$ (along dimension $i$ ) is denoted by $P_{n}=\left\{p_{n}\left(x_{i}\right)\right\}$, the tensor product for $d$ dimensions is given by the set

$$
P_{n}^{d}=\left\{p(x) \mid p(x)=\prod_{i=1}^{d} p_{n}\left(x_{i}\right) \text { for } p_{n}\left(x_{i}\right) \in P_{n}\right\} .
$$

For example, if $d=3$ and $n=1$ the set is given by

$$
P_{1}^{3}=\left\{c, x_{1}, x_{2}, x_{3}, x_{1} x_{2}, x_{1} x_{3}, x_{2} x_{3}, x_{1} x_{2} x_{3}\right\},
$$

and the total degree of the highest order polynomial in $P_{1}^{3}$ is 3 .
} 
number of unknown coefficients to be solved is $n^{d}$. This is the well-known curse of dimensionality.

The alternative we propose is to use 'Smolyak points' for the interpolation and to use linear combinations of polynomials which interpolate function values in certain directions. In order to understand the method, note that even in one dimension, in order to approximate a smooth function on the interval $[-1,1]$ by interpolating $n$ of its function values, one needs to carefully choose the nodes $x_{1}, \ldots, x_{n}$. It is known that both the extrema and the zeros of the Chebychev polynomials are nearly optimal in the following sense. Given any continuous function $f:[-1,1] \rightarrow \mathbb{R}$, let $g_{n}^{*}$ denote the polynomial of degree $n$ that minimizes $\max _{x \in[-1,1]}\left|f(x)-g_{n}^{*}(x)\right|$. Then if $p_{n}$ interpolates $f$ either at the $(n+1)$ Chebyvhev zeros or at the $(n+1)$ extrema, we have

$$
\max _{x \in[-1,1]}\left|p_{n}(x)-f(x)\right| \leq \Lambda_{n} \max _{x \in[-1,1]}\left|g_{n}^{*}(x)-f(x)\right|,
$$

with $\Lambda_{n} \leq C+\frac{2}{\pi} \log (n+1)$, where $C$ is independent of $n$ and the bounds are sharp, in the sense that they attained for some (classes of) examples. (see e.g. Cheney and Light (2000)).

Following Barthelmann et al. (2000), we use the extrema of Chebychev polynomials as our basis for the grid of points $\mathcal{H}$. We let $\mathcal{G}^{1}=\{0\}$ and for $n=2, \ldots$, we define the sets $\mathcal{G}^{n}=\left\{\zeta_{1}, \ldots, \zeta_{n}\right\} \subset[-1,1]$ as the set of the extrema of the Chebychev polynomials

$$
\zeta_{j}=-\cos \left(\frac{\pi(j-1)}{n-1}\right) \quad j=1, \ldots, n .
$$

Define a sequence of positive integers by $m(1)=1$ and $m(i)=2^{i-1}+1$ for $i=2,3, \ldots$. It is easy to see, and this turns out to be crucial for Smolyak's construction, that the sets of interpolation points satisfy $\mathcal{G}^{m(i)} \subset \mathcal{G}^{m(i+1)}$ for all $i$. The construction of the integers $m(i)$ is therefore crucial in assuring that the sets of Chebychev extrema are nested, as long as only sets of size $m(i)$ are permitted.

Smolyak's method uses this fact to build a hierarchical sparse grid out of combinations of the grids $\mathcal{G}^{m(i)}$ for different values of $i$. We first present a simple, albeit still abstract, three-dimensional example to illustrate the intuition of this idea and then move to a general description of the method.

\subsubsection{The Three-Dimensional Case}

We choose three dimensions because we can represent our selection of grid points graphically, and two dimensions are not sufficient to clarify how exactly the method avoids the curse of dimensionality.

We want to approximate a smooth function $f:[-1,1]^{3} \rightarrow \mathbb{R}$ by a polynomial of relatively small degree with few monomial terms, and we are looking for a method that is flexible in the sense that it is easy to add terms of higher degree and thereby increase the quality of the approximation. 
Define a 3-dimensional grid of approximation level $\lambda \geq 1$ as follows:

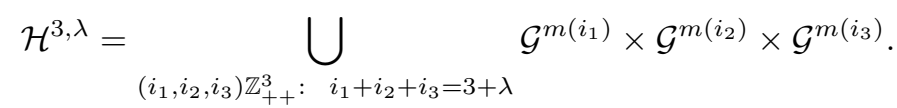

Recall that for $m(i)$ as defined above, the grids $\mathcal{G}^{m(i)}$ are nested for $i=$ $1,2, \ldots$. In order to understand how the formula constructs the interpolation points, it is useful to go through the cases $\lambda=1,2,3$ one by one. Recall that $\mathcal{G}^{m(1)}=\{0\}$ and that $\mathcal{G}^{m(2)}$ consists of three points, $\{-1,0,1\}$. Then, according to formula (10),

$\mathcal{H}^{3,1}=\mathcal{G}^{m(2)} \times \mathcal{G}^{m(1)} \times \mathcal{G}^{m(1)} \cup \mathcal{G}^{m(1)} \times \mathcal{G}^{m(2)} \times \mathcal{G}^{m(1)} \cup \mathcal{G}^{m(1)} \times \mathcal{G}^{m(1)} \times \mathcal{G}^{m(2)}$.

So the first level grid consists of the 7 points $(-1,0,0),(0,0,0),(1,0,0),(0,-1,0)$, $(0,1,0),(0,0,-1)$ and $(0,0,1)$.

Now, let's move to the case $\lambda=2$. Recall that $\mathcal{G}^{m(3)}$ consists of 5 points. The formula gives

$$
\begin{aligned}
\mathcal{H}^{3,2}=\mathcal{G}^{m(3)} & \times \mathcal{G}^{m(1)} \times \mathcal{G}^{m(1)} \cup \mathcal{G}^{m(1)} \times \mathcal{G}^{m(3)} \times \mathcal{G}^{m(1)} \cup \mathcal{G}^{m(1)} \times \mathcal{G}^{m(1)} \times \mathcal{G}^{m(3)} \\
\cup \mathcal{G}^{m(2)} & \times \mathcal{G}^{m(2)} \times \mathcal{G}^{m(1)} \cup \mathcal{G}^{m(2)} \times \mathcal{G}^{m(1)} \times \mathcal{G}^{m(2)} \cup \mathcal{G}^{m(1)} \times \mathcal{G}^{m(2)} \times \mathcal{G}^{m(2)}
\end{aligned}
$$

Figure 1 shows where these points are located in the three-dimensional cube $[-1,1]^{3}$. It first shows, for clarity, the point grids in two of the three dimensions, holding the third dimension fixed at 0 , that is, at $\mathcal{G}^{m(1)}$. The last panel then shows the three dimensional grid, which is generated as the appropriate union of the three two-dimensional sets (see formula (10)).

Note that $\mathcal{H}^{3,1} \subset \mathcal{H}^{3,2}$ and in general, as one can verify from Equation (10), $\mathcal{H}^{3, \lambda} \subset \mathcal{H}^{3, \lambda+1}$. Passing from level $\lambda$ to the next level of approximation $\lambda+1$ can therefore be viewed as adding new points to the existing grid.

At the risk of boring the reader, for clarification let us consider one more level of approximation, i.e. $\lambda=3$ which leads to $m(4)=9$ points along each dimension (holding the two others fixed at zero). We have as the third level Smolyak grid

$$
\begin{aligned}
& \mathcal{H}^{3,3}=\mathcal{G}^{m(4)} \times \mathcal{G}^{m(1)} \times \mathcal{G}^{m(1)} \cup \mathcal{G}^{m(1)} \times \mathcal{G}^{m(4)} \times \mathcal{G}^{m(1)} \cup \mathcal{G}^{m(1)} \times \mathcal{G}^{m(1)} \times \mathcal{G}^{m(4)} \\
& \cup \mathcal{G}^{m(3)} \times \mathcal{G}^{m(2)} \times \mathcal{G}^{m(1)} \cup \mathcal{G}^{m(2)} \times \mathcal{G}^{m(3)} \times \mathcal{G}^{m(1)} \\
& \cup \mathcal{G}^{m(3)} \times \mathcal{G}^{m(1)} \times \mathcal{G}^{m(2)} \cup \mathcal{G}^{m(2)} \times \mathcal{G}^{m(1)} \times \mathcal{G}^{m(3)} \\
& \cup \mathcal{G}^{m(1)} \times \mathcal{G}^{m(3)} \times \mathcal{G}^{m(2)} \cup \mathcal{G}^{m(1)} \times \mathcal{G}^{m(2)} \times \mathcal{G}^{m(3)} \cup \mathcal{G}^{m(2)} \times \mathcal{G}^{m(2)} \times \mathcal{G}^{m(2)}
\end{aligned}
$$

\subsubsection{Grids in Arbitrary Dimensions}

In general, for approximation in the d-dimensional hypercube, we can construct grids $\mathcal{H}^{d, \lambda}$ in exactly the same fashion. In order to give the general formula, for

arbitrary dimension $d$ and arbitrary approximation level $\lambda$, define a multi-index to be a vector of positive integers $\mathbf{i}=\left(i_{1}, \ldots, i_{d}\right) \in \mathbb{Z}_{++}^{d}$ and let $|\mathbf{i}|=i_{1}+\ldots+i_{d}$. 
Figure 1: Smolyak Points
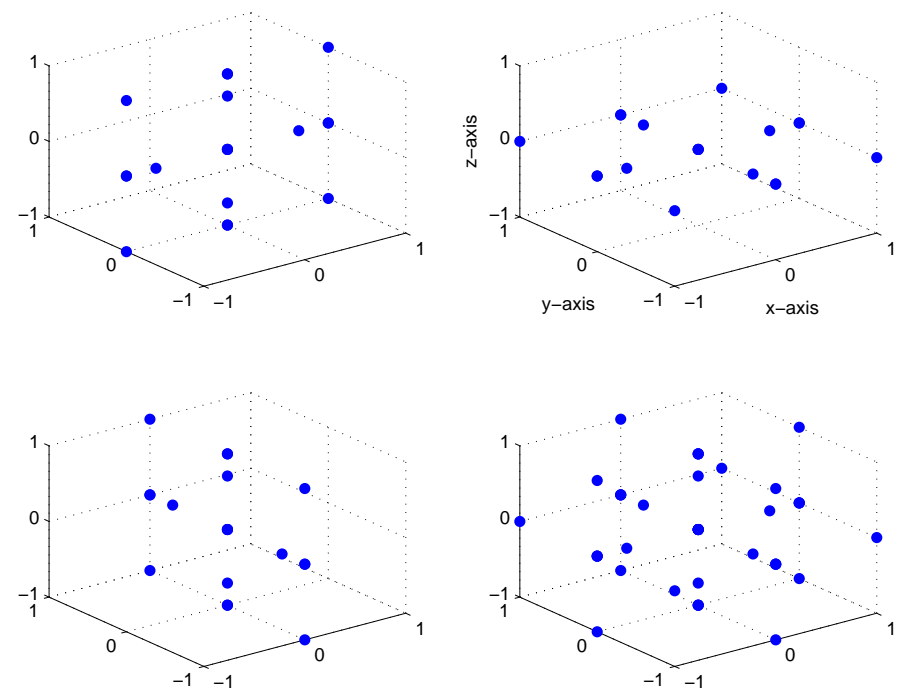

For integers $\lambda \geq 1$, we can then define a sparse grid of points in $[-1,1]^{d}$ as follows:

$$
\mathcal{H}^{d, \lambda}=\bigcup_{\mathbf{i}:|\mathbf{i}|=d+\lambda}\left(\mathcal{G}^{m\left(i_{1}\right)} \times \ldots \times \mathcal{G}^{m\left(i_{d}\right)}\right) .
$$

It can be easily verified that for $d=3$ and $\lambda=1,2,3$, this gives the sets of points which we described in the previous example.

It turns out that for the international real business cycle model solved in this paper, a level 2 construction is sufficient to obtain fairly high accuracy, so for arbitrary dimension $d$, we always consider $\mathcal{H}^{d, 2}$ as our set of points. Note that this is simply the union of $d$-dimensional sets of the form

$$
\begin{aligned}
& \mathcal{G}^{m(1)} \times \mathcal{G}^{m(1)} \times \ldots \times \mathcal{G}^{m(3)} \times \ldots \times \mathcal{G}^{m(1)} \\
& \mathcal{G}^{m(1)} \times \mathcal{G}^{m(2)} \times \ldots \times \mathcal{G}^{m(2)} \times \ldots \times \mathcal{G}^{m(1)}
\end{aligned}
$$

Since a complete enumeration of these sets is straightforward, the construction of $\mathcal{H}^{d, 2}$ is very simple for arbitrary dimension $d$.

\subsubsection{Interpolation}

Given the construction of these points, we now briefly describe an easy way to construct an interpolating polynomial. Smolyak's method interpolates at nodes in $\mathcal{H}$, using weighted sums of polynomials which interpolate subsets of $\mathcal{H}$.

Define $p^{\mathbf{i}}$ to be the tensor-product multivariate polynomial which interpolates on $\mathcal{G}^{m\left(i_{1}\right)} \times \ldots \times \mathcal{G}^{m\left(i_{d}\right)}$. As pointed out above, we represent this in Cheby- 
chev form (that is, use Chebychev polynomials), i.e.

$$
p_{\theta}^{\mathbf{i}}=\sum_{l_{1}=1}^{m\left(i_{1}\right)} \ldots \sum_{l_{d}=1}^{m\left(i_{d}\right)} \theta_{l_{1} \ldots l_{d}} T_{l_{1}} \ldots T_{l_{d}} .
$$

The coefficients $\theta_{l_{1} \ldots l_{d}}$ can be efficiently computed as follows. Consider a full $d$-dimensional grid with $k_{1}, \ldots, k_{d}>1$ points along each direction. Then

$\theta_{l_{1} \ldots l_{d}}=\frac{2^{d}}{\left(k_{1}-1\right) \cdots\left(k_{d}-1\right)} \frac{1}{c_{l_{1}} \cdots c_{l_{d}}} \sum_{j_{1}=1}^{k_{1}} \cdots \sum_{j_{d}=1}^{k_{d}} \frac{T_{l_{1}}\left(\zeta_{j_{1}}\right) \cdots T_{l_{d}}\left(\zeta_{j_{d}}\right) \cdot f\left(\zeta_{j_{1}}, \ldots, \zeta_{j_{d}}\right)}{c_{j_{1}} \cdots c_{j_{d}}}$

with $c_{1}=c_{k_{d}}=2$ and $c_{j}=1$ for $j=2, \ldots, k_{d}-1$. For the Smolyak construction, directions with only one point are then simply dropped, in the sense that if $m\left(i_{d}\right)=1$, we do not include the direction in the computation of the coefficient.

To return briefly to the three-dimensional example, $p^{(i, 1,1)}$ is the polynomial of degree $2^{i-1}$ which interpolates $m(i)=2^{i-1}+1$ points in the first direction and is constant along the second and third dimension. $p^{(2,2,1)}$ is the tensor product of two univariate polynomials of degree $m(2)-1=2$ and interpolates the function on the 3 by 3 grid $\mathcal{G}^{m(2)} \times \mathcal{G}^{m(2)} \times \mathcal{G}^{m(1)}$. For $\lambda=2$, it then might seem that the interpolating polynomial for the entire grid $\mathcal{H}^{3,2}$ should be a weighted sum of the univariate polynomials in each direction as well as the 2 -dimensional tensor product on each plane. However, it turns out that things are not quite as simple, and that one also needs to include the polynomials that interpolate $\mathcal{H}^{3,1}$. To see this, note that in order to interpolate the points in $\mathcal{G}^{m(2)} \times \mathcal{G}^{m(2)} \times \mathcal{G}^{m(1)}$, one would have to weight the polynomial $p^{2,2,1}$ with one and all others with zero. But then, to interpolate points in $\mathcal{G}^{m(3)} \times \mathcal{G}^{m(1)} \times \mathcal{G}^{m(1)}$ which are not in the previous grid (e.g. the point $(\cos (\pi / 4), 0,0))$ one would have to weight the polynomial $p^{3,1,1}$ with one. Of course, in order to do both, one would need to subtract some polynomials. The solution to this is to take the weighted sum not only of polynomials associated with $\mathcal{H}^{3,2}$ but also those associated with $\mathcal{H}^{3,1}$ (i.e. the polynomials $p^{2,1,1}, p^{1,2,1}, p^{1,1,2}$ ) as well as the constant $p^{1,1,1}$. It turns out that by including these, it is possible to find the correct weights to interpolate all points in $\mathcal{H}^{3,2}$.

In general, the Smolyak function which interpolates on $\mathcal{H}^{d, \lambda}$ is given by the weighted sum of low dimensional tensor products. Denote by $q=\max (d, \lambda+1)$. At a point $x \in[-1,1]^{d}$ we then approximate $f(x)$ by

$$
\hat{f}^{d, \lambda}(x)=\sum_{q \leq|\mathbf{i}| \leq d+\lambda}(-1)^{d+\lambda-|\mathbf{i}|}\left(\begin{array}{c}
d-1 \\
d+\lambda-|\mathbf{i}|
\end{array}\right) p^{\mathbf{i}}(x) .
$$

The weights, $(-1)^{d+\lambda-|\mathbf{i}|}\left(\begin{array}{c}d-1 \\ d+\lambda-|\mathbf{i}|\end{array}\right)$, are chosen to ensure that the weighted sum of polynomials which interpolate on subsets of $\mathcal{H}$ interpolates on the entire set. ${ }^{7}$

\footnotetext{
${ }^{7}$ See Barthelmann et al. (2000) for a proof that this procedure indeed works.
} 


\subsubsection{Some Properties of Smolyak's Method}

Without getting into mathematical details, we want to briefly discuss the advantages of Smolyak's method. The first obvious advantage is that the number of grid points does not grow exponentially with the dimension. It can be verified that the number of points in $\mathcal{H}^{d, \lambda}$ is $1+2 d$ for $\lambda=1,1+4 d+4 \frac{d(d-1)}{2}$ for

$\lambda=2$ (the level of approximation used in this paper), and $1+8 d+12 \frac{d(d-1)}{2}+$ $8 \frac{d(d-1)(d-2)}{6}$ for $\lambda=3$. The nestedness of the nodes, $\mathcal{G}^{m(i)}$, implies that in general the number of points in $\mathcal{H}^{d, \lambda}$ grows only polynomially in $d$ if $\lambda$ is taken fixed. Note that it grows quickly in $\lambda$, but the examples below show that very good approximations are achieved even for $\lambda=2$.

Moreover, it can be shown that $\hat{f}^{d, \lambda}$ exactly replicates any polynomial function of degree less than or equal to $\lambda$, in the sense that $\hat{f}^{d, \lambda}$ is identically equal to this polynomial if $\hat{f}^{d, \lambda}$ interpolates it at the Smolyak points. At a first glance this might seem a bit disappointing. After all, $\hat{f}^{d, \lambda}$ is a polynomial of degree $2^{\lambda}$ - however, since the ratio between the degree of $\hat{f}$ and the degree of any polynomial that can be replicated by $\hat{f}$ is independent of the dimension $d$, the algorithm is regarded as nearly optimal. In general, better schemes are not known. Moreover, this makes clear that using a level-2 Smolyak approximation is at least as good as (and often strictly better than) using any second degree polynomial.

\subsection{Integration}

Once we approximate the unknown policy functions by Smolyak polynomials, we require that the unknown coefficients ensure that equations (1)-(4) hold exactly at the points in the grid. In order to solve for the coefficients, we obviously need a way to evaluate the integral in equation (1). Since $u_{c}$ and $f_{k}$ as well as the probability density function are not polynomials, we need to approximate the integral numerically. It is well known that for integration in relatively low dimensions (say around 10-15), if the integrand is sufficiently smooth, routines based on interpolatory cubature rules turn out to deliver much more accurate results than Monte Carlo or quasi Monte Carlo methods (see Cools (2002) or Schürer (2003)). Since Judd's (1998) textbook contains an excellent description of these various rules we do not discuss them in detail here. In the computations, we use a degree 5 rule for an integrand on an unbounded range weighted by a 
standard normal. ${ }^{8}$

Note that although the exogenous shocks $(a)$ in our model are not normally distributed, we can easily rewrite the integral in equation (1) as a function of the underlying innovations $(\varepsilon)$ which are indeed standard normal. Thus, the integral is in the form $\int_{\mathbb{R}^{d}} f(x) e^{-\sum_{i=1}^{d} x_{i}^{2}} d x$ and can be approximated by this degree 5 rule. ${ }^{9}$

\subsection{Finding the Unknown Coefficients}

Using Smolyak's polynomials to approximate the policy functions and using the cubature rule to approximate the integrals now allows us to consider a finite system of non-linear equations whose solutions are the unknown coefficients $\theta$ of the approximate policy-functions. In principle, this system can be solved easily by modern non-linear equation solvers which are variations of Newton's method. However, since the focus of this paper lies on Smolyak's method to construct sparse grids, and since we want to be able to easily increase the size of the problem (by adding additional countries and hence additional endogenous and exogenous states), we chose to solve for the coefficients by a "time-iteration" algorithm.

First we guess policy functions $K_{0}^{i \prime}(s), L_{0}^{i}(s)$ and $C_{0}^{i}(s)$ for all $i \in I$. For a given iteration $n-1$ and associated policy functions $\left\{K_{n-1}^{i \prime}(s), L_{n-1}^{i}(s), C_{n-1}^{i}(s)\right\}_{i \in I}$ the iteration $n$ policy functions are, for all $s=(k, a)$, defined by the $3 N$ equa-

\footnotetext{
${ }^{8}$ More precisely,

$\int_{\mathbb{R}^{d}} f(x) e^{-\sum_{i=1}^{d} x_{i}^{2}} d x \approx A f(0)+B \sum_{i=1}^{d}\left(f\left(r e^{i}\right)+f\left(-r e^{i}\right)\right)+$ $D \sum_{i=1}^{d-1} \sum_{j=i+1}^{d}\left(f\left(s e^{i}+s e^{j}\right)+f\left(s e^{i}-s e^{j}\right)+f\left(-s e^{i}+s e^{j}\right)+f\left(-s e^{i}-s e^{j}\right)\right)$,
}

with

$$
r=\sqrt{1+d / 2}, s=\sqrt{1 / 2+d / 4}, A=\frac{2 \pi^{d / 2}}{d+2}, B=\frac{(4-d) \pi^{d / 2}}{2(d+2)^{2}}, D=\frac{\pi^{d / 2}}{(d+2)^{2}} .
$$

\footnotetext{
${ }^{9}$ In order to verify the quality of approximation, we compared the results with a simple Monte-Carlo method that uses 10000 draws. In all cases, the differences were on the order of at most $10^{-5}$.
} 
tions $^{10}$

$$
\begin{aligned}
& \tau^{i} u_{c}^{i}\left(C_{n}^{i}(s), L_{n}^{i}(s)\right) \\
& =\frac{\beta \int \tau^{i} u_{c}^{i}\left(C_{n-1}^{i}\left(s^{\prime}\right), L_{n-1}^{i}\left(s^{\prime}\right)\right)\left[\begin{array}{c}
1+a^{i \prime} f_{k}^{i}\left(K_{n}^{i \prime}(s), L_{n-1}^{i}\left(s^{\prime}\right)\right)+ \\
\frac{\phi}{2} \frac{\left(K_{n-1}^{i \prime}\left(s^{\prime}\right)-K_{n}^{i \prime}(s)\right)\left(K_{n-1}^{i \prime}\left(s^{\prime}\right)+K_{n}^{i \prime}(s)\right)}{K_{n}^{i \prime}(s)^{2}}
\end{array}\right] g_{a}\left(a^{\prime}\right) d a^{\prime}}{1+\frac{\phi\left(K_{n-1}^{i \prime}\left(s^{\prime}\right)-K_{n}^{i \prime}(s)\right)}{K_{n}^{i \prime}(s)}} \\
& =\frac{\beta \int \tau^{i} u_{c}^{i}\left(C_{n-1}^{i}\left(s^{\prime}\right), L_{n-1}^{i}\left(s^{\prime}\right)\right)\left[\begin{array}{c}
1+a^{j \prime} f_{k}^{j}\left(K_{n}^{i \prime}(s), L_{n-1}^{j}\left(s^{\prime}\right)\right)+ \\
\frac{\phi}{2} \frac{\left(K_{n-1}^{j \prime}\left(s^{\prime}\right)-K_{n}^{j \prime}(s)\right)\left(K_{n-1}^{j \prime}\left(s^{\prime}\right)+K_{n}^{j \prime}(s)\right)}{K_{n}^{j \prime}(s)^{2}}
\end{array}\right] g_{a}\left(a^{\prime}\right) d a^{\prime}}{1+\frac{\phi\left(K_{n-1}^{j \prime}\left(s^{\prime}\right)-K_{n}^{j \prime}(s)\right)}{K_{n}^{j \prime}(s)}} \\
& =\tau^{j} u_{c}^{j}\left(C_{n}^{j}(s), L_{n}^{j}(s)\right) \\
& \begin{aligned}
\frac{u_{l}^{i}\left(C_{n}^{i}(s), L_{n}^{i}(s)\right)}{u_{c}^{i}\left(C_{n}^{i}(s), L_{n}^{i}(s)\right)} & =-a^{i} f_{l}^{i}\left(k^{i}, L_{n}^{i}\right) \\
C_{n}(s)+\sum_{i=1}^{N} K_{n}^{i \prime}(s)+\sum_{i=1}^{N} \frac{\phi\left(K_{n}^{i \prime}(s)-k^{i}\right)^{2}}{2 k^{i}} & =Y(s)+K .
\end{aligned}
\end{aligned}
$$

In terms of running-times, this method is obviously not comparable to Newton's method, however, it has the advantage that it can easily handle very large systems. Moreover, it has a nice economic interpretation in that it can be viewed as approximating the infinite horizon economy by an economy with a large finite horizon.

\section{Numerical Results}

We present results - including policy functions plots, approximation errors, and running times - for a number of specifications of the model described in the previous section. Throughout, we approximate the policy functions with a polynomial of total degree 4 (i.e., $\lambda=2$ ). Higher-order approximations yield smaller approximation errors at the cost of longer running times.

\subsection{Model Specifications}

The various model specifications we solve differ by the number of countries $N$, the forms of the utility and production functions, and the parameter values chosen for the technology shock process, capital adjustment costs, and utility and production functions. "Problem A" of the JEDC Numerical Methods Comparison Project (Den Haan, Judd, and Juillard (2004)) provides a complete

\footnotetext{
${ }^{10}$ Again we abuse notation and let $s^{\prime}=\left(K_{n}^{\prime}(s), a^{\prime}\right)$.
} 
description of the different specifications and their calibration. Following is a condensed version of this description.

As described above, the capital adjustment cost is assumed to be quadratic with scale parameter $\phi$. The period utility function is of one of the forms ${ }^{11}$

$$
u(c, l)=\left\{\begin{array}{c}
\frac{c^{1-\frac{1}{\gamma}}}{1-\frac{1}{\gamma}} \\
\frac{c^{1-\frac{1}{\gamma}}}{1-\frac{1}{\gamma}}-b \frac{l^{1+\frac{1}{\eta}}}{1+\frac{1}{\eta}} \\
\frac{\left(c^{\psi}\left(L^{e}-l\right)^{(1-\psi)}\right)^{1-\frac{1}{\gamma}}}{1-\frac{1}{\gamma}} \\
\frac{\left[\left(c^{1-\frac{1}{\chi}}+b\left[L^{e}-l\right]^{1-\frac{1}{\chi}}\right)^{\frac{1}{1-\frac{1}{\chi}}}\right]^{1-\frac{1}{\gamma}}}{1-\frac{1}{\gamma}},
\end{array}\right.
$$

and the production function is of one of the forms

$$
f(k, l)=\left\{\begin{array}{c}
A\left(\alpha k^{\mu}+(1-\alpha) l^{\mu}\right)^{\frac{1}{\mu}}, \mu \neq 0 \\
A k^{\alpha} l^{1-\alpha}, \mu=0 .
\end{array}\right.
$$

Table 1 lists the parameters which vary across specifications and the functional forms used in each specification. ${ }^{12}$

\subsubsection{Challenges for Solving the Model}

A few key issues arise in the application of Smolyak's method, described generally in Section 3, to our specific economic model. The first issue is choosing bounds for the state variables, a necessary step since Smolyak's method is defined over a closed hypercube, $[-1,1]^{2 N} \cdot{ }^{13}$ For the exogenous state variables, we simply set $\left[\ln \left(\underline{a}^{i}\right), \ln \left(\bar{a}^{i}\right)\right]=\left[\frac{-t r \sigma}{1-\rho}, \frac{\operatorname{tr} \sigma}{1-\rho}\right]$, where $\operatorname{tr}$ is some positive scalar. For the endogenous state variable, the bounds must be chosen with great care to ensure the capital policy functions, $K^{n \prime}(k, a)$, stay within the chosen bounds. All else equal, the wider the capital bounds the more likely the capital policy function is to stay in bounds but the poorer the accuracy of the solution.

There is also an interaction between the bounds on capital and the bounds on the technology shocks. As is known from the one-country stochastic growth model, capital exhibits a positive, hump-shaped response to a technological shock, and thus, for a fixed capital interval, larger technological shocks make it more likely for the capital policy function to go out of bounds. For this reason,

\footnotetext{
${ }^{11}$ The case $\gamma=1$ is understood to be the logarithmic case.

${ }^{12}$ The other parameter values are $\beta=0.99, \delta=0.025, \alpha=0.36$, and $A=(1-\beta) /(\alpha \beta)$. $A$ is chosen so the steady-state capital stock is equal to unity for all countries, and (where applicable) $b$ and $\psi$ are chosen so the steady-state labor supply is also equal to unity. The value of $L^{e}=2.5$ is chosen so that steady-state labor supply equals $40 \%$ of time endowment, $L^{e}$. Finally, the Pareto weights $\left(\tau^{1}, \ldots, \tau^{N}\right)$ are pinned down by assuming that each country optimally consumes its net output in steady state.

${ }^{13}$ Although the state variable generally does not lie in $[-1,1]^{2 N}$, it lies in the box $\mathcal{B}=$ $\left[\underline{k}^{1}, \bar{k}^{1}\right] \times \ldots \times\left[\underline{k}^{N}, \bar{k}^{N}\right] \times\left[\ln \left(\underline{a}^{1}\right), \ln \left(\bar{a}^{1}\right)\right] \times \ldots \times\left[\ln \left(\underline{a}^{N}\right), \ln \left(\bar{a}^{N}\right)\right]$. It is straightforward to use a change of variables to map a state $x \in \mathcal{B}$ to $[-1,1]^{2}$.
} 
Table 1: Model Specifications

\begin{tabular}{lccccccc}
\hline \hline Mod & N & Volatility & $\phi$ & $\gamma$ & $\eta$ & $\chi$ & $\mu$ \\
\hline A1 & 2,6 & L, H & $0.5,10$ & 1 & - & - & 0 \\
A2 & 2,6 & L, H & $0.5,10$ & $0.25,1$ & $0.1,1$ & - & 0 \\
A3 & 2,6 & L, H & $0.5,10$ & $0.25,1$ & - & - & 0 \\
A4 & 2,6 & L, H & $0.5,10$ & 0.25 & - & 0.83 & -0.2 \\
A5 & 2,6 & L, H & $0.5,10$ & $(0.25,1)$ & - & - & $(0,0)$ \\
A6 & 2,6 & L, H & $0.5,10$ & $(0.25,1)$ & $(0.1,1)$ & - & $(0,0)$ \\
A7 & 2,4 & L, H & $0.5,10$ & $(0.5,1)$ & - & - & $(0,0)$ \\
A8 & 2,4 & L, H & $0.5,10$ & $(0.2,0.4)$ & - & $(0.75,0.9)$ & $(-0.3,0.3)$ \\
\hline
\end{tabular}

Notes: As described in Den Haan, Judd, and Juillard (2004), A1 and A5 have inelastic labor supply with CRRA utility over consumption; A2 and A6 have separable utility functions with isoleastic labor supply and CRRA utility over consumption; A3 and A7 exhibit Cobb-Douglas utility over consumption and leisure, while A4 and A8 have CES utility. In A1-A4, the countries are symmetric, while in $\mathrm{A} 5-\mathrm{A} 8$, the parameters of the utility and production functions differ across countries. An entry $(x, y)$ for a given parameter $\zeta$ indicates that country $i=1, \ldots, N$ has parameter $\zeta_{i}=x+\frac{i-1}{N-1}(y-x)$. Finally, low (L) volatility corresponds to $\rho=0.8, \sigma=0.001$ and high $(\mathrm{H})$ volatility to $\rho=0.95, \sigma=0.01$.

the high volatility case tends to be more difficult to solve than the low volatility case, but both can be solved with the proper choice of bounds. Unless noted otherwise, we use $\operatorname{tr}=1.25$ and $[\underline{k}, \bar{k}]=\left[0.5 k_{s s}, 1.5 k_{s s}\right]$ to generate all the results for the symmetric cases (A1-A4) reported below.

For asymmetric cases with endogenous labor supply (A6-A8), finding appropriate bounds for the capital interval provides an additional challenge because the true capital policy functions are asymmetric across countries. To see this, consider specifications A6-A8 without any capital adjustment costs $(\phi=0)$ or technology shocks $\left(\ln \left(a_{t+1}^{i}\right)=0\right)$. In equilibrium, the marginal utility of consumption is equalized across countries at each date, and thus one can see from the intertemporal Euler equations that the distribution of $t+1$ capital will be set to equate the marginal product of capital across countries in $t+1$, which requires equating countries' capital/labor ratios. Because some countries have more elastic labor supplies than others, the countries will supply different levels of labor if provided with the same non-steady-state capital stock. Figure 4 (to be described later) illustrates this for specification A6. Consequently, the true equilibrium features asymmetric capital policy functions. Solving for these policy functions thus requires specifying asymmetric bounds for the country-specific capital stocks. ${ }^{14}$ In practice, we let $\left[\underline{k}_{1}, \bar{k}_{1}\right]=\left[0.42 k_{s s}, 1.58 k_{s s}\right]$,

\footnotetext{
${ }^{14}$ The program converges when using symmetric capital intervals (for example, symmetric intervals were used to create Figures $2-4$ ), but the accuracy is better in the case of asymmetric capital intervals.
} 
$\left[\underline{k}_{N}, \bar{k}_{N}\right]=\left[0.58 k_{s s}, 1.42 k_{s s}\right]$, and choose intermediate values for the widths of the other countries' capital intervals. ${ }^{15}$

A second issue that arises in using Smolyak's method is finding a good initial guess for the time-iteration algorithm to compute policy functions. In general it is not possible to establish that the operator used for the time-iteration procedure is a contraction mapping, and thus, convergence is not guaranteed for any initial guess. In fact, it turns out that poor guesses for labor supply often lead to difficulties for our method. To minimize these difficulties, we have at times found it useful to slightly alter the time-iteration algorithm described in Section 3.3. Rather than using the previous iteration of the policy function for labor supply $L_{n-1}\left(s^{\prime}\right)$ on the right-hand side of the intertemporal Euler equation, we solve for tomorrow's labor, denoted by $L^{*}$, using

$$
\frac{u_{l}^{i}\left(C_{n-1}^{i}\left(s^{\prime}\right), L^{*}\right)}{u_{c}^{i}\left(C_{n-1}^{i}\left(s^{\prime}\right), L^{*}\right)}=-a^{i} f_{l}^{i}\left(K_{n}^{i \prime}(s), L^{*}\right) .
$$

This reduces the importance of the initial guess for labor supply, which turns out to be especially helpful for finding a solution in cases A6-A8.

Finally, our procedure can solve specifications of the model with up to $N=6$ countries (12 state variables). As currently written, our procedure does not exploit any of the symmetries between the countries (for cases A1-A4) when computing the solution of the model. But because the Smolyak points are symmetric (see Figure 1), one could envision utilizing the symmetry of cases A1-A4 by solving for the policy functions of only one country at each iteration and then doing the proper transformation to generate the policy functions of the other countries. Doing so may make solving for $N=10$ countries less time intensive in these cases. However, given the results reported below, in which increasing the number of countries does not appear to lead to particularly interesting economic insights, we choose not to pursue this direction of research at this point.

\subsubsection{Policy Functions}

Figures 2 - 4 plot the country-specific capital, consumption, and labor policy functions for specification A6 with $N=2$, high volatility, and low adjustment costs. There are four plots in each figure: the plots show the policy functions of both countries (blue-solid $=$ country 1 , red-dashed $=$ country 2 ) as a function of the own country's and the other country's capital stocks (top two plots) and own country's and other country's technology shock (bottom two plots), holding the other state variables constant at their steady-state values. ${ }^{16}$

These policy functions are representative, in a qualitative sense, of the policy functions from other specifications. In particular, the capital policy functions

\footnotetext{
${ }^{15}$ For case $\mathrm{A} 8$, the order of the capital grids is reversed with country $\mathrm{N}$ having the widest grid, i.e., $\left[\underline{k}_{1}, \bar{k}_{1}\right]=\left[0.58 k_{s s}, 1.42 k_{s s}\right],\left[\underline{k}_{N}, \bar{k}_{N}\right]=\left[0.42 k_{s s}, 1.58 k_{s s}\right]$.

${ }^{16}$ In order to show all the interesting movements in the policy functions, we solved over a capital interval of $\left[0.1 k_{s s}, 1.9 k_{s s}\right]$ and set $t r=1.25$ so the technological shock interval is $[-0.25,0.25]$.
} 
are fairly linear in all state variables, while consumption is slightly concave with respect to capital and slightly convex with respect to technology shocks. The labor supply functions clearly display the most significant non-linearities, with labor supply being a non-monotonic function of a country's own capital stock. We take these non-linearities as evidence that non-linear solution methods do, in fact, provide better approximations of the true solution of the model than linear methods. Kim, Kim, and Kollmann (2007) (KKK) document this same result quantitatively by showing that a quadratic approximation of the solution outperforms a linear approximation. Our solution method is also significantly more accurate than a linear approximation, as can be seen by comparing our results reported below (in Table 2 ) to the linear approximation results in KKK.

It is also interesting to note the effect of asymmetric parameter values on the policy functions. The labor supply functions again display the most interesting results. The optimality condition for labor supply of country $i$ can be written as

$$
l_{t}^{i}=\left[\frac{A^{1 / \gamma^{1}} a_{t}^{i} k_{t}^{i^{\alpha}}}{c_{t}^{1 / \gamma^{1}}}\right]^{\frac{\eta^{i}}{1+\eta^{i} \alpha}},
$$

where we have substituted in the optimality condition equating the marginal utility of consumption across countries. ${ }^{17}$ Thus, it is easy to see that country $2\left(\eta^{2}=1.0\right)$ will have a more elastic labor supply with respect to a movement in productivity than country $1\left(\eta^{1}=0.1\right)$, as is confirmed by Figure 4 . It is also interesting to note that, except at very low capital levels, labor supply falls with an increase in own-country capital because the direct income effect (i.e., increased consumption) overwhelms the substitution effect from a higher wage (top-left panel of Figure 4). Furthermore, consumption is also more elastic for country $2\left(\gamma^{2}=1.0\right)$ than country $1\left(\gamma^{1}=0.25\right)$ because the equilibrium condition for consumption-sharing implies that $c_{t}^{2}$ is proportional to $\left(c_{t}^{1}\right)^{\gamma^{2} / \gamma^{1}}$.

\subsection{Approximation Errors and Running Times}

We check the accuracy of the solution in three ways, the first two of which require the computation of conditional error functions. These functions, denoted by $R_{n}\left(x_{t}\right)$ for $n=1, . ., 3 N$, are unit-free versions of the $3 N$ optimality conditions (see equations (1) - (4)) evaluated at the present state $x_{t} \equiv\left(k_{t}, a_{t}\right)$. The true solution of the model has $R_{n}\left(x_{t}\right)=0$, for all $n$ and $x_{t}$. These functions are

\footnotetext{
${ }^{17}$ Note that the Pareto weights are $\tau_{i}=\frac{1}{u_{c}^{i}\left(c_{s s}^{i}, l_{s s}^{i}\right)}=A^{1 / \gamma^{i}}$.
} 
Figure 2: Capital Policy Functions for A6
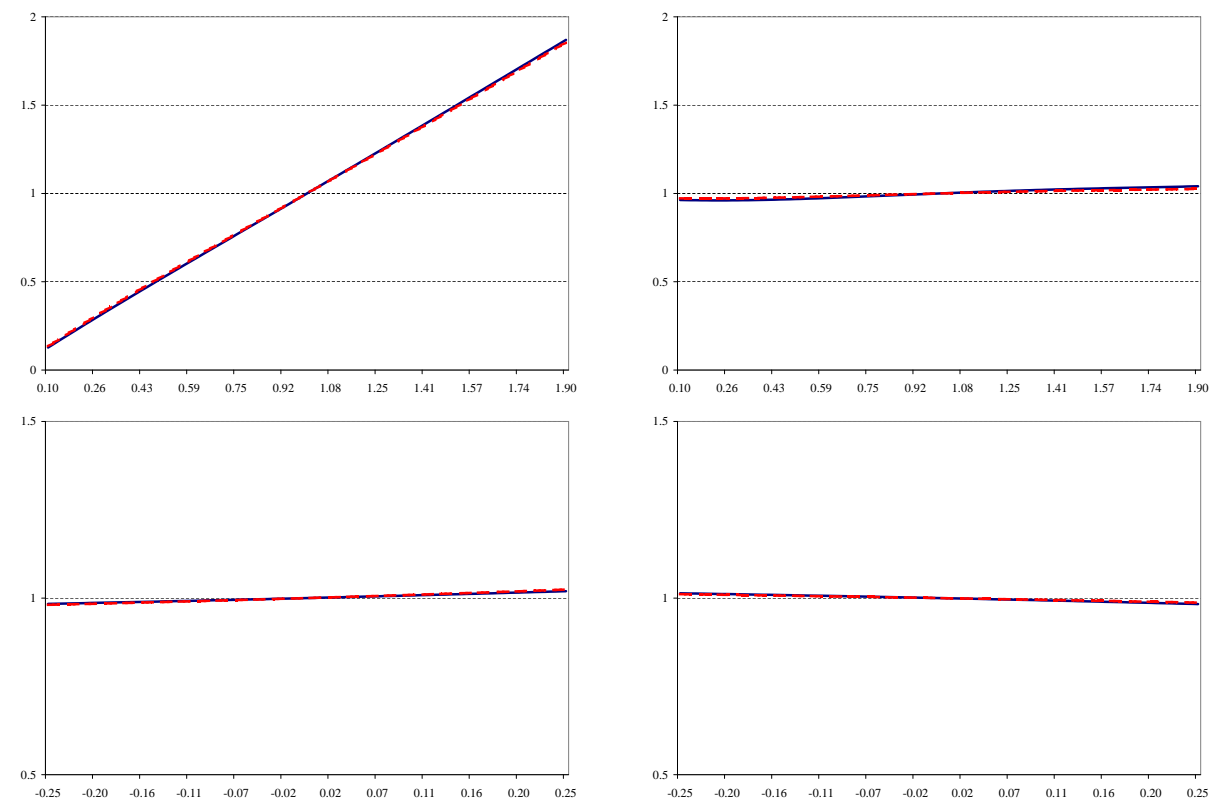

Notes: Capital stock tomorrow as a function of own and other capital stocks (top two plots) and own and other technology shocks (bottom two plots), holding other state variables at steady-state values. The blue lines are for country 1 $(\gamma=0.25, \eta=0.1)$, and the red-dashed lines are for country $2(\gamma=1.0, \eta=1.0)$. Model specification: A6, $N=2$, high volatility, $\phi=0.5$. 
Figure 3: Consumption Policy Functions for A6
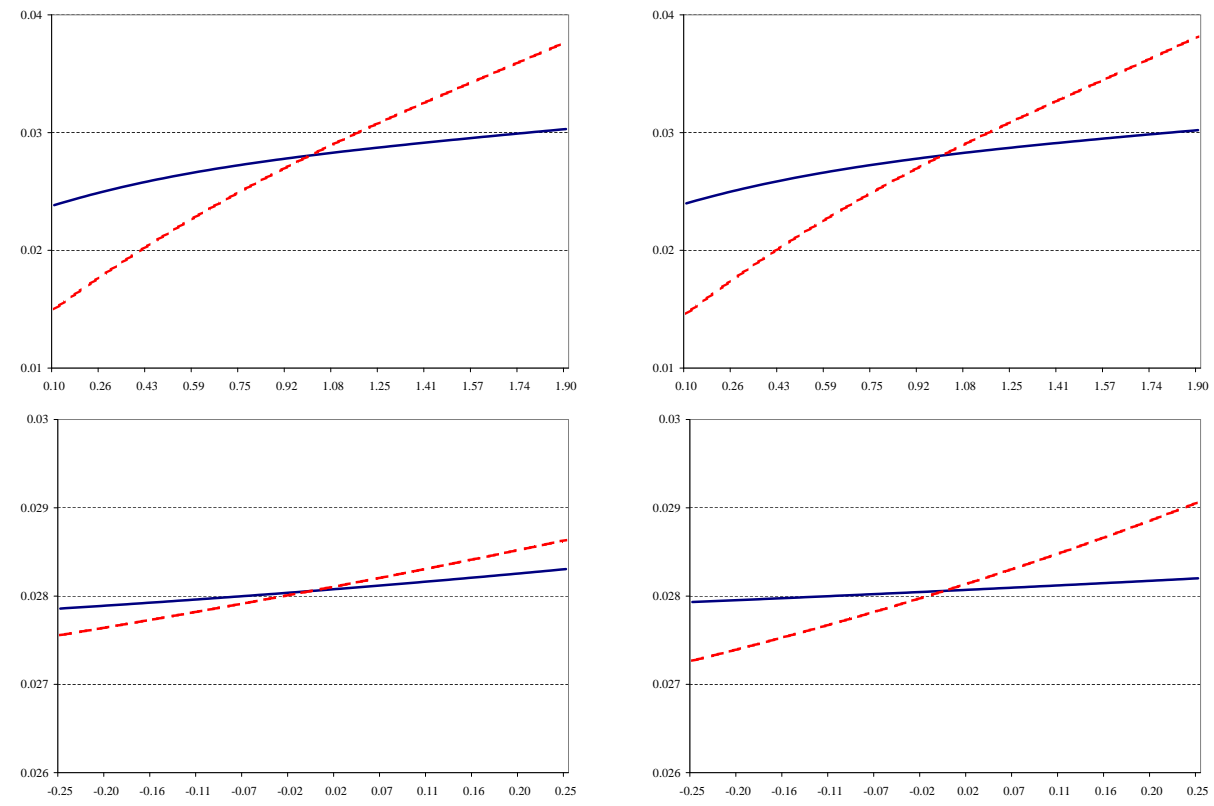

Notes: Consumption as a function of own and other capital stocks (top two plots) and own and other technology shocks (bottom two plots), holding other state variables at steady-state values. The blue lines are for country $1(\gamma=$ $0.25, \eta=0.1)$, and the red-dashed lines are for country $2(\gamma=1.0, \eta=1.0)$. Model specification: A6, $N=2$, high volatility, $\phi=0.5$. 
Figure 4: Labor Policy Functions for A6
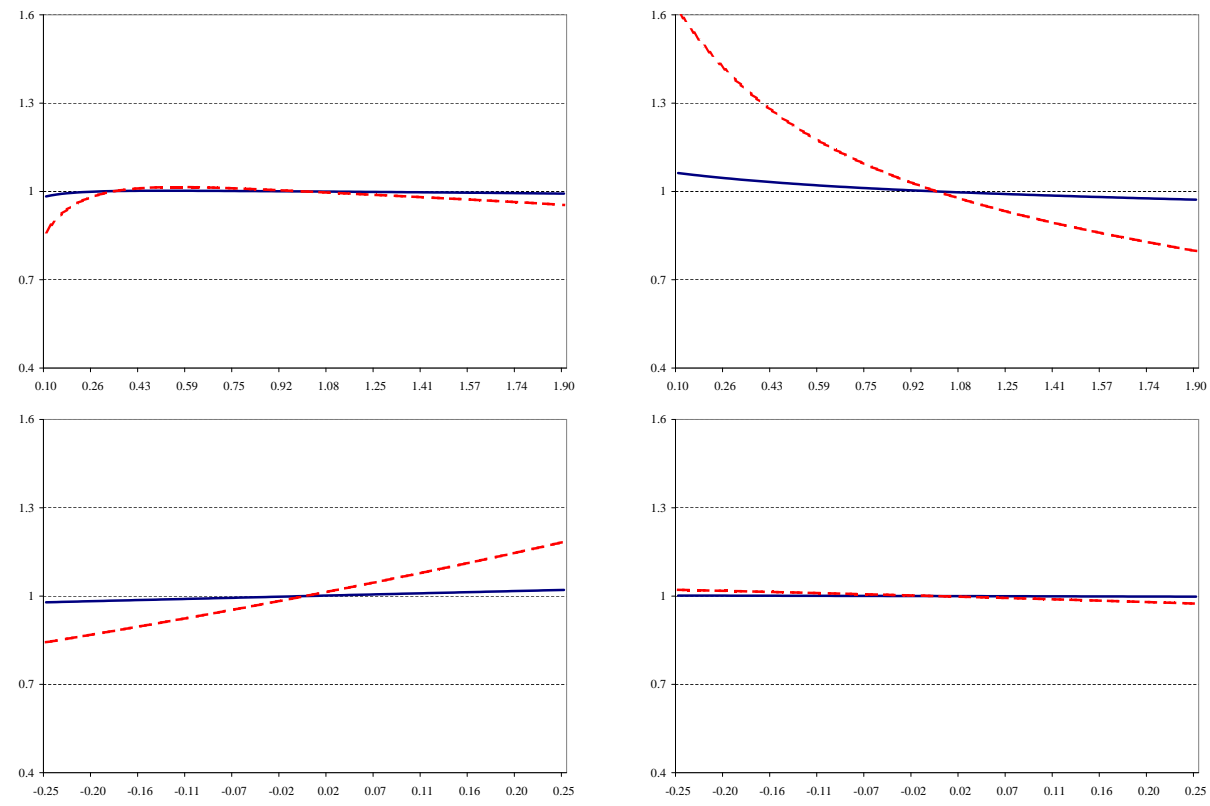

Notes: Labor supply as a function of own country and other country capital stocks (top two plots) and own and other technology shocks (bottom two plots), holding other state variables at steady-state values. The blue lines are for country $1(\gamma=0.25, \eta=0.1)$, and the red-dashed lines are for country 2 $(\gamma=1.0, \eta=1.0)$. Model specification: A6, $N=2$, high volatility, $\phi=0.5$. 
given by

$$
\begin{aligned}
R_{n} & \equiv \beta E_{t}\left\{\frac{u_{c, t+1}^{n}}{u_{c, t}^{n}} \frac{\left[1+a_{t+1}^{n} f_{k, t+1}^{n}+\phi_{2, t+1}^{n}\right]}{\left[1+\phi_{1, t}^{n}\right]}\right\}-1, \text { for } n=1, \ldots, N \\
R_{n} & \equiv \frac{u_{c, t}^{n-N} a_{t}^{n-N} f_{l, t}^{n-N}}{u_{l, t}^{n-N}}+1, \text { for } n=N+1, \ldots, 2 N \\
R_{2 N+1} & \equiv \frac{Y_{t}+K_{t}}{C_{t}+K_{t+1}+\phi_{t}}-1 \\
R_{n} & \equiv \frac{\tau^{n-2 N} u_{c, t}^{n-2 N}}{\tau^{1} u_{c, t}^{1}}-1, \text { for } n=2 N+2, \ldots, 3 N
\end{aligned}
$$

where the arguments of all functions have been suppressed, e.g., $u_{c, t}^{n} \equiv u_{c}^{n}\left(c^{n}\left(x_{t}\right), l^{n}\left(x_{t}\right)\right) .{ }^{18}$ Then, letting $R\left(x_{t}\right)$ denote the $3 N$-dimensional vector of conditional errors evaluated at state, $x_{t}$, Accuracy Tests 1 and 2 are implemented as follows:

Accuracy Test 1: $R\left(x_{t}\right)$ is computed for 100 independent random vectors $x_{t}=\left\{k_{t}^{i}, a_{t}^{i}\right\}_{i=1}^{N}$ at radius $r$ from the deterministic steady-state, for $r=\{0.01,0.02,0.05,0.10,0.15,0.20,0.30\} .{ }^{19}$ We report $T_{r} \equiv \max _{n, t}\left|R_{n}\left(x_{t}\right)\right|$.

Accuracy Test 2: The model is simulated for 1000 periods. ${ }^{20}$ Let $S_{n, \max } \equiv \max _{t}\left|R_{n}\left(x_{t}\right)\right|$ and $S_{n, \text { mean }} \equiv \operatorname{mean}\left(\left|R_{n}\left(x_{t}\right)\right|\right)$ for $t=1, \ldots, 1000$. We report $S_{\text {max }}$ and $S_{\text {mean }}$, which are the maximum $S_{n, \text { max }}$ and $S_{n, \text { mean }}$ (across $n)$.

The third accuracy test is the so-called Den Haan-Marcet statistic (Den Haan and Marcet (1994)) and focuses solely on the $N$ intertemporal equilibrium conditions. This statistic tests whether the realized errors in the intertemporal conditions are orthogonal to a constant and first- and second-order monomials of the state variables. This holds for the true solution since the intertemporal equilibrium conditions are conditional expectations. Accuracy Test 3 is implemented as follows:

Accuracy Test 3: We run 200 simulations of the model, each lasting 1000 periods. For each run, the Den Haan-Marcet statistic (1994, p.5) is constructed, and we compare the frequency distribution of this statistic (across the 200 simulations) to the theoretical $\chi_{N\left(2 N^{2}+3 N+1\right)}^{2}$ distribution, where the degrees of freedom for the $\chi^{2}$ distribution are determined by the number of

\footnotetext{
${ }^{18}$ We use the degree- 5 monomial integration formula described in Section 3.2 to calculate the expectations in the intertemporal conditions.

${ }^{19}$ Recall that our solution method requires us to place bounds on the state variables. The bounds for the capital stocks are roughly 0.5 units from the steady state, while those for the technology shocks are 0.25 units away $(t r=1.25, \rho=0.95, \sigma=0.01)$. Thus, it is possible that a sampled point $r=0.30$ units from the steady state could lie outside the technology shock bound for one country. In this case, we reduce the deviation from the steady state in that dimension and increase the deviations equally in all other dimensions. In effect, we choose a different sample point that is still $r=0.30$ units from the steady-state and also in bounds.

${ }^{20}$ The state variables are initially set at their steady state values. The actual length of the simulation was 1200 periods, and the first 200 periods were discarded to ensure independence from initial conditions. We use this same 'burn-off' period for Accuracy Test 3.
} 
intertemporal equilibrium conditions $(N)$ multiplied by the number of instruments $\left(2 N^{2}+3 N+1\right)$. We report the percentage of the simulations with a statistic in the lower $\left[P_{.05}\right]$ and upper $\left[P_{.95}\right]$ critical $5 \%$ of the $\chi^{2}$ distribution. Fairly similar distributions are taken as evidence for an accurate solution.

Table 2 reports results of the accuracy tests for 20 of the 96 specifications listed in Table 1. For A2 and A3, we only report results for the parameterizations with greatest and least curvature since these results bracket the others. All reported statistics are for high volatility, $\rho=0.95$ and $\sigma=0.01$, and low adjustment costs $\phi=0.5$ because these cases usually ${ }^{21}$ have the largest conditional error functions. Increasing the adjustment costs to $\phi=10$ typically reduces the errors slightly, while lowering the volatility has a much larger effect. In fact, of all parameters, those with the biggest impact on the solution accuracy are the volatility parameters.

The numbers shown for Tests 1 and 2 are logs of the error measures $\left(\log _{10}\left(T_{r}\right)\right.$, $\left.\log _{10}\left(S_{\text {max }}\right), \log _{10}\left(S_{\text {mean }}\right)\right)$. Recall that the true solution has conditional error functions equal to zero, and thus, a smaller reported statistic implies greater accuracy. It is clear from the results of Test 1 that the accuracy of our approximation is highest when the economy is close to its steady state, as $T_{r}$ is smallest when $r$, the distance from the steady state, is small. This fact also helps explain why the errors reported for Accuracy Test 2 lie between the errors for $T_{.01}$ and $T_{.3}$; in the simulations we ran for Accuracy Test 2 , the state variables $[k, \ln (a)]$ always lie within 0.12 of their steady-state values.

One can also see from Table 2 that the number of countries $N$ typically does not have a large impact on the error measures $T_{r}, S_{\text {max }}$, and $S_{\text {mean }}$, although in cases A4 and A6, the decline in accuracy with higher $N$ is more substantial. The curvature parameters have some impact on accuracy as specifications with more curvature (low $\eta$ and/or low $\gamma$ for A2-A3) have larger approximation errors close to the deterministic steady-state $\left(T_{.01}\right)$ but smaller approximation errors further away $\left(T_{.30}\right)$. Finally, functional forms A3/A7 and A4/A8 appear to have slightly larger approximation errors than A1/A5 and A2/A6, although this may have as much to do with differences in curvature (parameter values) as it does with the particular functional forms.

Although we only report the largest errors across all conditional error functions, it turns out that these almost always correspond to the intertemporal Euler equations. This deserves some comment. In our solution procedure, the static conditions that determine labor supply and the sharing of consumption across countries hold quite exactly. This is because we solve these equations as functions of the state variables and consumption of country 1, without imposing any functional form on the labor supply of any country or the consumption of countries $2-N$. Thus, even though the policy functions for labor supply may be

\footnotetext{
${ }^{21}$ The high volatility case of a particular specification always has larger errors (Accuracy Test 2) than the low volatility case, while the low adjustment cost case usually does. In the instances when high adjustment costs produce larger errors, the difference is never greater than 0.25 (in $\log _{10}$ ). None of our qualitative conclusions hinge importantly on reporting results for $\phi=0.5$ rather than $\phi=10$.
} 
highly nonlinear (see top-left panel of Figure 4), they do not present a problem for our solution method. Rather, any approximation errors will occur mainly in the intertemporal Euler equations and aggregate resource constraint.

Table 2: Accuracy tests: results for selected specifications

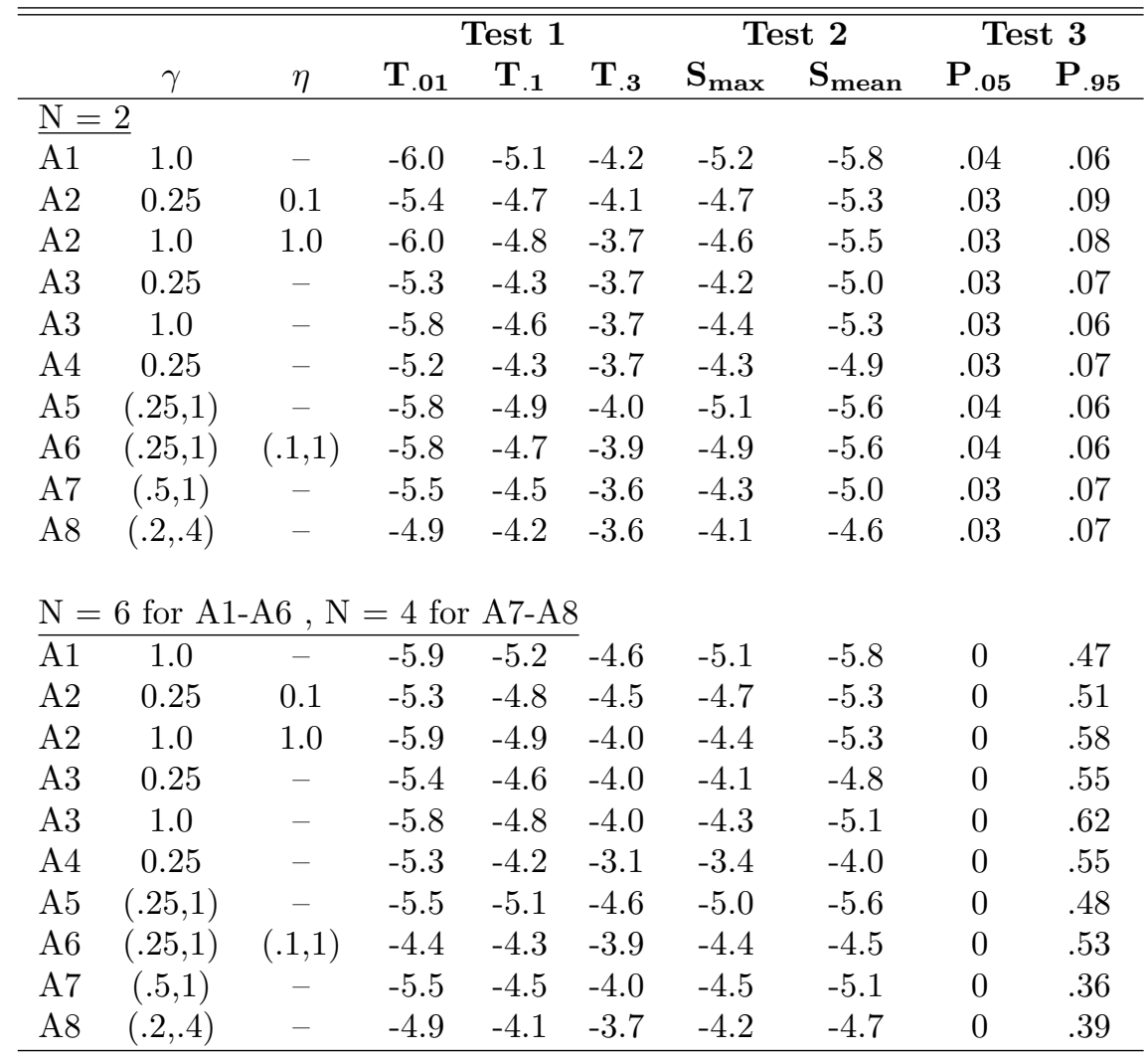

Notes: The first three columns specify the model and some parameters that vary across alternative specifications. All reported statistics are for high volatility, $\rho=0.95$ and $\sigma=0.01$, and low adjustment $\operatorname{costs} \phi=$ 0.5. The figures shown for Tests 1 and 2 are logs of the error measures $\left(\log _{10}\left(T_{r}\right), \log _{10}\left(S_{\max }\right), \log _{10}\left(S_{\text {mean }}\right)\right)$.

For Test 3, recall that numbers near or below .05 provide evidence of an accurate solution. Thus, the Den Haan-Marcet accuracy measures in Table $2^{22}$ are much worse when the number of countries is large $(N=4,6)$ than when

\footnotetext{
${ }^{22} \mathrm{As}$ mentioned earlier, the reported measures in Table 2 are for the high volatility and low adjustment cost case. The Den Haan-Marcet measures suggest our solution method is less accurate in the high adjustment cost case $(\phi=10)$, with approximately $13 \%$ of the test statistics lying in the upper critical $5 \%$ of the $\chi^{2}$ distribution for $N=2$. Results available upon request.
} 
$N=2$ : the percentage of observations of the test statistic in the upper critical $5 \%$ of the $\chi^{2}$ distribution is much greater than $5 \%$ for large $N$. At this point, it is not clear whether these measures reflect poor accuracy of our solution method or sensitivity of the DM statistic to a large number of instruments. In our tests, there are 15 instruments when $N=2,45$ when $N=4$, and 91 when $N=6$, whereas the largest number of instruments used by Den Haan and Marcet (1994) was 7. Further experiments will be done to assess whether Accuracy Test 3 is, in fact, a good accuracy measure for this class of problems.

Table 3 reports the time required to compute and run accuracy tests on the solutions of different specifications of the model. ${ }^{23}$ In particular, the column labelled "Sol" shows the time it takes to compute the solution. For $N=2$ countries, this is on the order of seconds; for $N=4$ (not reported), it takes minutes; and for $N=6$, the program can take an hour or more.

Some specifications, namely A7 and A8, take significantly longer to run than others. For these specifications, our solution procedure, as described earlier, solves a nonlinear equation for the labor supply on the right-hand side of the intertemporal Euler equation rather than simply using the labor supply policy function from the previous iteration. Because this must be done quite often, the program takes much more time to converge, and we choose to only solve for policy functions for up to $N=4$ countries for these specifications. We also solve a nonlinear equation for labor in specification $\mathrm{A} 6$, but because it is possible to solve the equation analytically in this case, the program runs relatively quickly.

\section{Conclusion}

In this paper we described and used a projection method based on Smolyak's algorithm to compute globally accurate solutions to models featuring a sizeable number of continuous state variables. The method was applied to solving a wide variety of international real business cycle model specifications with high accuracy and reasonable running times and appears to be a viable solution method for use in a wide class of economic models.

One goal of this paper has been to make this solution method more accessible to other economists. To this end, we provided both a general description of the method and a discussion of some of the practical challenges to implementing it, such as choosing appropriate bounds for the state variables. We have made available corresponding computer code for the Smolyak method, ${ }^{24}$ which should enable any researcher armed with a set of optimality conditions to solve for the nonlinear solution of her model without having to undertake the fixed costs of implementing the construction of the Smolyak grid.

\footnotetext{
${ }^{23}$ These programs were run using FORTRAN 6.6 on a Pentium 4 PC $(2.8 \mathrm{GHz})$.

${ }^{24}$ The program for specification A8 of this paper is available at http://www.econ.upenn.edu/ ${ }^{\sim}$ dkrueger/research.php.
} 
Table 3: Computing Times for selected models

\begin{tabular}{lcccccc}
\hline \hline & & & \multicolumn{4}{c}{ Time(seconds) } \\
\cline { 4 - 7 } & $\gamma$ & $\eta$ & Sol. & Test 1 & Test 2 & Test 3 \\
\hline \multicolumn{1}{c}{$\mathrm{N}=2$} & & & & & & \\
$\mathrm{~A} 1$ & 1.0 & - & 1.0 & 0.01 & 0.07 & 1.9 \\
$\mathrm{~A} 2$ & 0.25 & 0.1 & 1.7 & 0.02 & 0.08 & 2.2 \\
$\mathrm{~A} 2$ & 1.0 & 1.0 & 1.5 & 0.02 & 0.08 & 2.2 \\
$\mathrm{~A} 3$ & 0.25 & - & 6.2 & 0.7 & 1.0 & 14.6 \\
$\mathrm{~A} 3$ & 1.0 & - & 4.8 & 0.7 & 1.0 & 14.2 \\
$\mathrm{~A} 4$ & 0.25 & - & 5.1 & 0.8 & 1.2 & 16.9 \\
$\mathrm{~A} 5$ & $(.25,1)$ & - & 1.3 & 0.01 & 0.06 & 1.9 \\
$\mathrm{~A} 6$ & $(.25,1)$ & $(.1,1)$ & 2.6 & 0.02 & 0.08 & 2.1 \\
$\mathrm{~A} 7$ & $(.5,1)$ & - & 73 & 0.7 & 1.1 & 14.6 \\
$\mathrm{~A} 8$ & $(.2, .4)$ & - & 70 & 0.8 & 1.2 & 16.9 \\
& & & & & & \\
$\mathrm{~N}=6$ & for A1-A6, N & 4 for & $\mathrm{A} 7-\mathrm{A} 8$ & & \\
$\mathrm{~A} 1$ & 1.0 & - & 1364 & 1.6 & 2.4 & 974 \\
$\mathrm{~A} 2$ & 0.25 & 0.1 & 2068 & 1.7 & 2.6 & 990 \\
$\mathrm{~A} 2$ & 1.0 & 1.0 & 1676 & 1.7 & 2.6 & 977 \\
$\mathrm{~A} 3$ & 0.25 & - & 5430 & 16.7 & 24.8 & 1210 \\
$\mathrm{~A} 3$ & 1.0 & - & 3244 & 16.4 & 24.4 & 1215 \\
$\mathrm{~A} 4$ & 0.25 & - & 3297 & 19.9 & 28.9 & 1039 \\
$\mathrm{~A} 5$ & $(.25,1)$ & - & 1718 & 1.6 & 2.4 & 1148 \\
$\mathrm{~A} 6$ & $(.25,1)$ & $(.1,1)$ & 2068 & 1.8 & 2.7 & 1201 \\
$\mathrm{~A} 7$ & $(.5,1)$ & - & 2949 & 4.9 & 7.3 & 64 \\
$\mathrm{~A} 8$ & $(.2, .4)$ & - & 2603 & 5.7 & 8.5 & 70 \\
\hline
\end{tabular}

Notes: Column labelled "Sol." is the computing time for the solution of the model, while the columns labelled "Test 1", "Test 2", and "Test 3" record the computing time for the various accuracy tests. All reported statistics are for high volatility, $\rho=0.95$ and $\sigma=0.01$, and low adjustment $\operatorname{costs} \phi=0.5$. 


\section{References}

[1] Backus, D., P. Kehoe and F. Kydland (1992), "International Real Business Cycles," Journal of Political Economy, 100, 745-775.

[2] Backus, D., P. Kehoe and F. Kydland (1995), "International Business Cycles, Theory and Evidence," in T. Cooley (ed.) Frontiers of Business Cycle Research, Princeton University Press, Princeton, NJ.

[3] Barthelmann, V., E. Novak and K. Ritter (2000), "High Dimensional Polynomial Interpolation on Sparse Grids", Advances in Computational Mathematics, 12, 273-288.

[4] Bungartz, H.J. and M. Griebel (2004), "Sparse grids ", Acta Numerica, 13: 147-269.

[5] Cheney, E. W. and W. Light (1999), A Course in Approximation Theory, Brooks/Cole Company.

[6] Cools, R., (2002), "Advances in multidimensional integration", Journal of Computational and Applied Mathematics, 149, 1-12.

[7] Den Haan, W., K. Judd, and M. Juillard (2004), "Comparing Numerical Solutions of Models with Heterogenous Agents," Mimeo, LBS, Stanford University, and CEPREMAP.

[8] Den Haan, W. and A. Marcet (1994), "Accuracy in Simulations," Review of Economic Studies, 61, 3-17.

[9] Judd, K. (1998), Numerical Methods in Economics, MIT Press, Cambridge, MA.

[10] Kim, J., S. Kim, and R. Kollmann (2007), "Solving a Multi-Country RBC Model Using Sims' Second-Order Accurate Algorithm," Mimeo, University of Paris XII.

[11] Krueger, D. and F. Kubler (2004), "Computing Equilibrium in OLG Models with Stochastic Production," Journal of Economic Dynamics and Control, 28(7), 1411-1436.

[12] Schürer, R. (2003), "A comparison between (quasi-) Monte Carlo and cubature rule based methods for solving high-dimensional integration problems" Mathematics and Computers in Simulation, 62, 509-517.

[13] Smolyak, S. (1963), "Quadrature and Interpolation Formulas for Tensor Products of Certain Classes of Functions" Soviet Mathematics, Doklady, 4, 240-243. 